\title{
Lung Ultrasound in Critically Ill Patients
}

\author{
Jinwoo Lee, M.D. \\ Division of Pulmonary and Critical Care Medicine, Department of Internal Medicine, Seoul National University College of Medicine, Seoul, Korea
}

Lung ultrasound (LUS) is an emerging tool for intensivists to diagnose and monitor thoracic diseases of critically ill patients. It is easily applied at the bedside in real time and is free of radiation hazards. In the intensive care units (ICUs) lung ultrasound can be used to diagnose pneumothorax and interstitial syndrome. It can also be used to monitor changes in the lung. However, the major limitations of LUS is that it is highly operator dependent and cannot be applied in patients with thoracic dressings, subcutaenous emphysema or pleural calcifications. This article reviews the basic principles of lung ultrasound and discusses how it can be used in ICUs.

Key Words: intensive care units; lung ultrasound.

\section{Introduction}

Thoracic imaging is routinely performed for most of patients in intensive care units (ICUs). One of the most easily accessible imaging modalities is a portable standard chest X-ray (CXR). However, regular CXR exams may be uncomfortable for critically ill patients and introduce potential hazards of irradiation. The CXR procedure, from examination request to final report, requires a considerable amount of time. The quality of the CXR image is often highly variable. Critically ill patients may also have multiple radiographic abnormalities that make CXR interpretation difficult.

A chest computed tomography (CT) is another frequently used diagnostic tool. For a chest CT scan, however, the patient needs to be transported to the radiology department, and critically ill patients are at increased risk of morbidity and mortality during transport.[1] Also, chest CT exposes patients to a substantial radiation dose, which limits the repeatability of the exam.[2]

For these reasons, the possibility of examining the lungs of critically ill patients using bedside ultrasound is gaining popularity among intensivists. This thoracic imaging modality complements conventional radiographic tools and is easily applied at the bedside in real time and is free of radiation hazards. Recently, consensus statements by an international panel of experts at the first International Consensus Conference on Lung Ultrasound (LUS) were published to provide evidence-based recommendations on point-of-care LUS.[3] The aim of this review is to describe the basic principles of LUS and present how it can be clinically applied in ICUs.

Received on December 30, 2015 Revised on February 16, 2016 Accepted on February 24, 2016

Correspondence to: Jinwoo Lee, Division of Pulmonary and Critical Care Medicine, Department of Internal Medicine, Seoul National University College of Medicine, 101 Daehak-ro, Jongno-gu, Seoul 03080, Korea

Tel: +82-2-2072-2114, Fax: +82-2-762-9662

E-mail: realrain7@gmail.com

*No potential conflict of interest relevant to this article was reported.

\section{Equipment and Examination Techniques}

Ideally, ultrasound machines should be portable, lightweight and able to store images and videos. A microconvex probe is preferred because it allows the probe to be positioned between the ribs to minimize shadows. However, other

(cc) This is an Open Access article distributed under the terms of the Creative Commons Attribution Non-Commercial License (http:/creativecommons.org/ licenses/by-nc/3.0/) which permits unrestricted non-commercial use, distribution, and reproduction in any medium, provided the original work is properly cited. Copyright (c) 2016 The Korean Society of Critical Care Medicine 
Table 1. Commonly used terminologies in lung ultrasound

\begin{tabular}{ll}
\hline Terminology & Movement of visceral pleura against parietal pleura during respiration. May exclude pneumothorax \\
\hline Lung sliding & Reverberation artifacts in normal lung \\
\hline A-lines (Fig. 2) & $\begin{array}{l}\text { Discrete laser-like vertical hyperechoic reverberation artifacts that arise from the pleural line. The number of these vertical B-lines } \\
\text { depends on the degree of lung aeration loss }\end{array}$ \\
$\begin{array}{ll}\text { B-lines, comet tail artifacts, } \\
\text { lung rockets (Fig. 3) }\end{array}$ & Massive loss of lung aeration. Hypoechoic tissue structure \\
Consolidation (Fig. 4) & Small movement of the visceral on the parietal pleura induced by heartbeat. Implications of intact pleural interface \\
\hline Lung pulse &
\end{tabular}

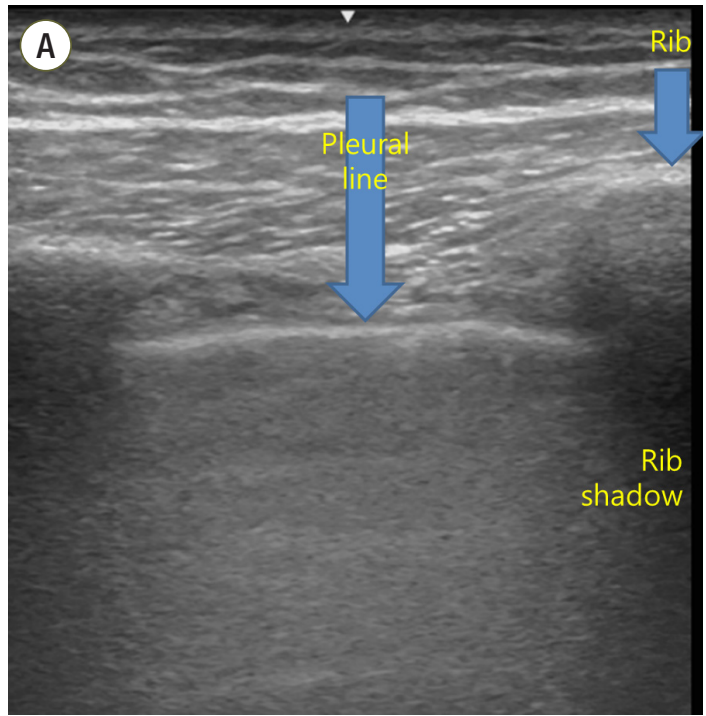

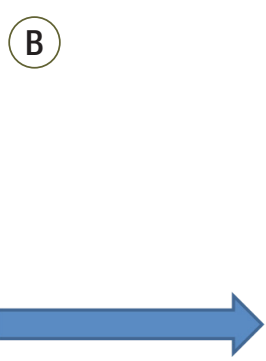

Pleural line

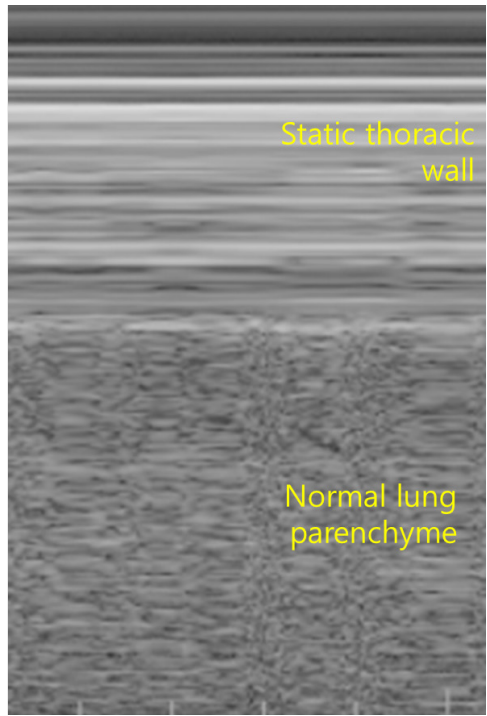

Fig. 1. Normal findings of lung ultrasound. (A) Characteristic upper-rib, pleural-line, lower-rib landmark that should be identified before starting the exam. (B) Normal lung pattern on M-mode: seashore sign. Motionless chest wall generates horizontal lines and lung sliding makes the sandy pattern below.

transducers such as linear (vascular), phased array (cardiac), convex (abdominal) probes may be used based on physician preferences and clinical settings.

Patients in the ICU are usually examined in a supine or semirecumbent position with arms abducted to facilitate examination of the lateral chest wall. Eight basic regions are assessed. Each hemithorax is divided into anterior and lateral regions by the anterior and posterior axillary lines. The regions are further divided into upper and lower areas. $[3,4]$ The posterior axillary line can be used as an anamical landmark to divide the hemithorax into 6 regions instead of 4 , allowing the assessment of the dependent lungs.[5,6]

\section{Principles of LUS}

LUS shows lung images that are based on both real im- ages and artifacts. In normal air-filled lungs, ultrasound waves cannot be transmitted. However, ultrasound findings can still be assessed in normal lungs and pleura. Furthermore, pathological conditions that decrease aeration of the lungs and other pleural abnormalities can be easily detected by LUS. The key factor in interpreting LUS is that it offers real-time dynamic images of the aeration state of the lung parenchyma. A decrease in aeration may be from "loss of air" (atelectasis) or by "accumulation of fluid or cells in the interstitial or alveolar space" (e.g., pneumonia, edema, contusion).[7] Commonly used LUS terminologies are in Table 1.

\section{Normal Findings}

The ultrasound probe should be placed longitudinally and perpendicular to the ribs on the chest wall. This allows iden- 


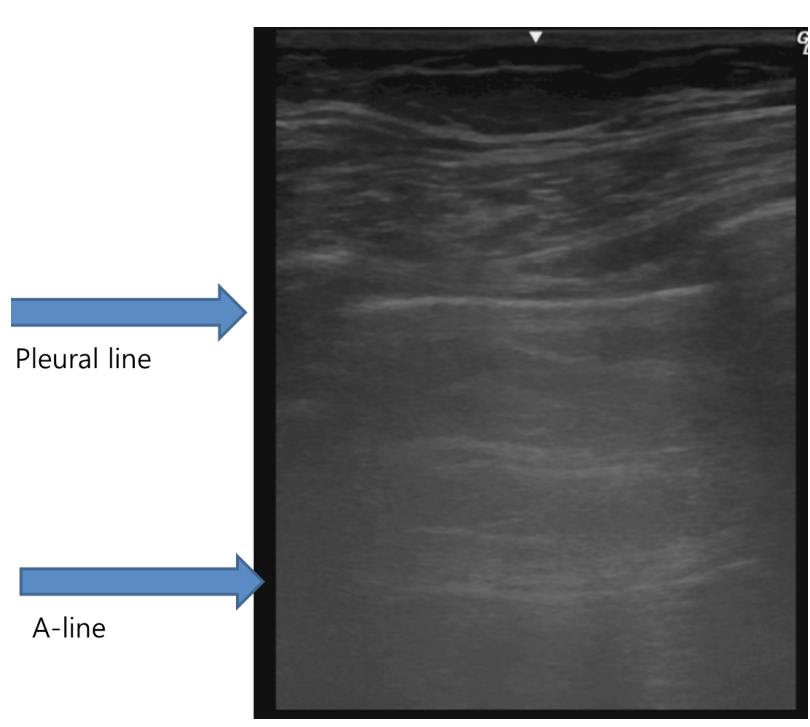

Fig. 2. Normal findings of lung ultrasound: A-lines. A-lines are horizontal hyperechoic lines parallel to the pleural line and are basic artifacts of a normally aerated lung.

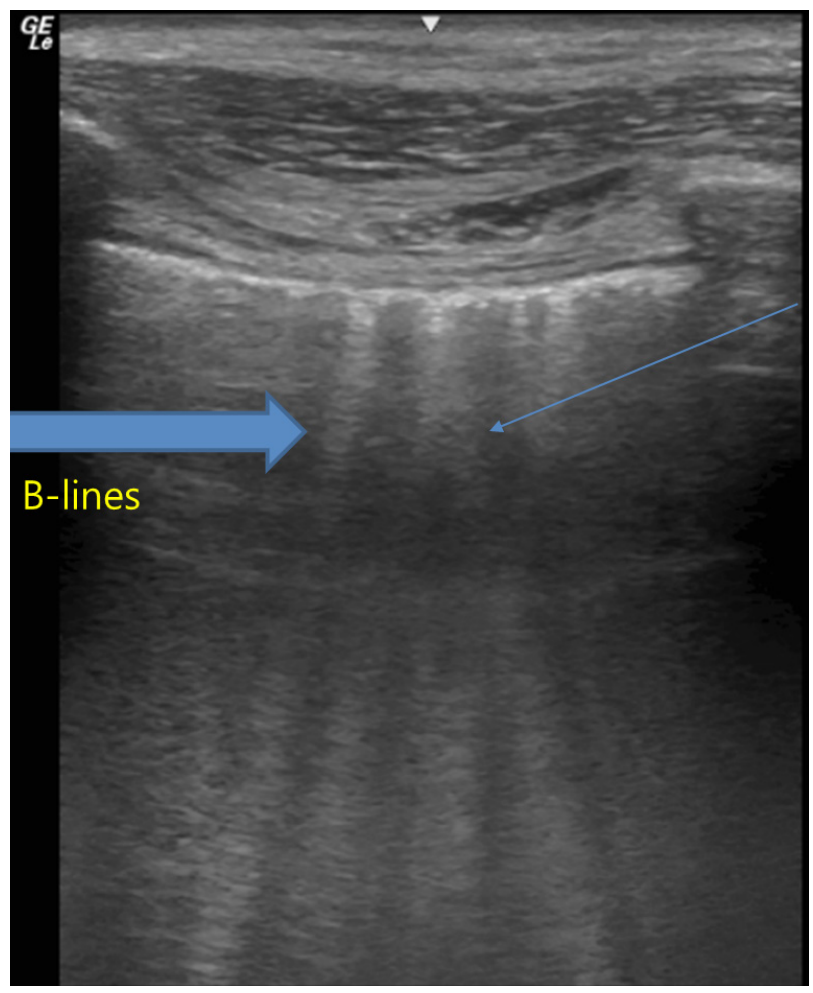

Fig. 3. B-lines. B-lines are hyperechoic vertical lines that arise from pleural lines extending to the bottom of the screen without fading and moving synchronously with lung sliding.

tification of the pleural line, ribs, and posterior shadowing beneath the ribs (Fig. 1A). Lung sliding is seen when the visceral pleura moves over the parietal pleura. Sonographi- cally, lung sliding is seen as a hyperechoic line that moves with respiration. This sign implies that that the visceral pleura is in contact with the parietal pleura and therefore excludes the possibility of pneumothorax. In M-mode images, lung sliding is seen as the "seashore sign" (Fig. 1B), with the static chest wall as waves and the normal lung parenchyma as a beach. This sign highlights the difference between the moving pleura and the motionless chest wall.

Beyond the pleural line, normally aerated lungs are seen as artifacts. Motionless, horizontal white lines regularly spaced that are equally distant between the skin and the pleural line that resemble the pleural line are reverberation artifacts. These artifacts are produced by echoes bouncing between the pleural line and the probe and are called A-lines. (Fig. 2). Although A-lines constitute a basic artifact of the normally aerated lung, they are also seen in pneumothorax without lung sliding.

\section{Pathological Findings}

\section{1) B-lines}

B-lines are hyperechoic lines perpendicular to the pleural lines extending to the bottom of the screen without fading and moving synchronously with lung sliding (Fig. 3). The number of B-lines depends on the degree of lung aeration loss. Their number increases with decreasing air content.[8,9] The presence of multiple B-lines is the sonographic sign of lung interstitial syndrome. A positive region is defined by the presence of three or more B-lines in a longitudinal plane between two ribs. Fewer than two B-lines can be detected in dependent lung regions even in normally aerated lungs.[10]

\section{2) Consolidation}

In contrast to normally aerated lungs, lung consolidation appears as a poorly defined hypoechoic or tissue-like structure, depending on the extent of air loss and the air-fluid ratio. (Fig. 4). Among the causes of lung consolidations are infection, pulmonary embolism, malignancy, contusion, atelectasis, and acute respiratory distress syndrome (ARDS).[3]

Air bronchograms are punctiform or linear hyperechoic artifacts within the consolidation. Dynamic air bronchogram, which is the respiratory movement of gas bubbles within the bronchus, indicates pneumonia and helps with 


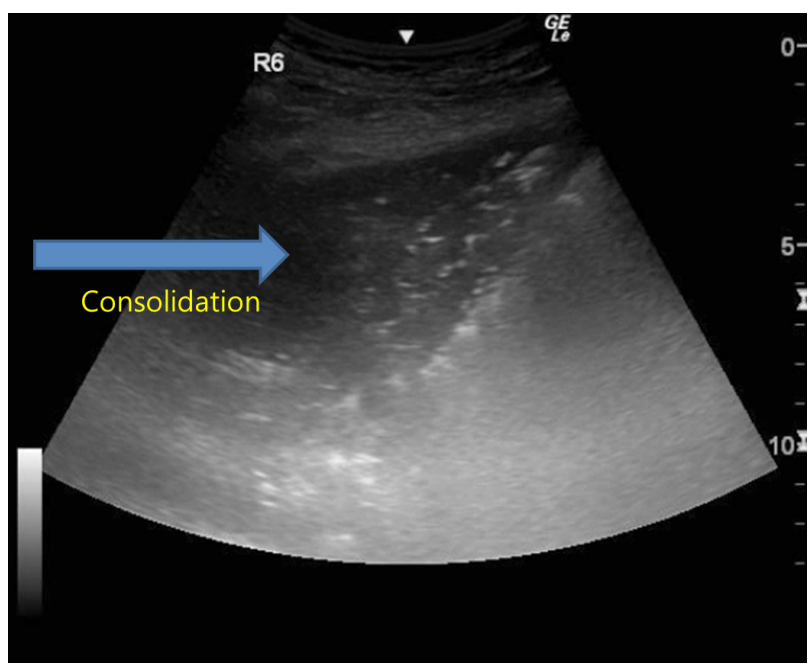

Fig. 4. Sonographic appearance of lung consolidation. Consolidation is sonographically observed as an area of tissue density. Within the consolidation, hyperechoic foci might be seen that represent air bronchograms.

differentiation from atelectasis.[11]

\section{Clinical Application in the ICU}

\section{1) Pneumothorax}

Pneumothorax is a potentially lethal complication of mechanical ventilation, thoracentesis and central-vein catheter insertion. To assess a patient in a supine position, the anterior chest, which is the least gravitationally dependent area, should be explored first followed by the lateral chest. No lung sliding should be seen and in M mode, only horizontal lines should be seen because dynamic lung sliding is absent. This characteristic pattern is called the stratosphere sign (Fig. 5). The presence of lung sliding effectively rules out pneumothorax with high negative predictive value of $99.2 \%$ to $100 \%$.[12,13] However, the absence of lung sliding is not specific for pneumothorax. This characteristic is also present in pathological conditions such as ARDS, atelectasis, pleural adhesions, pulmonary fibrosis and single-lung intubation.[14] To diagnose pneumothorax by LUS, B-lines and lung pulse (Table 1) should be should not be observed along with absent lung sliding. Also, the thorax should be scanned to find the lung point,[3] which is the point of transition where between visible lung sliding and the absence of lung sliding. This point represents the boundaries of the pneumo-

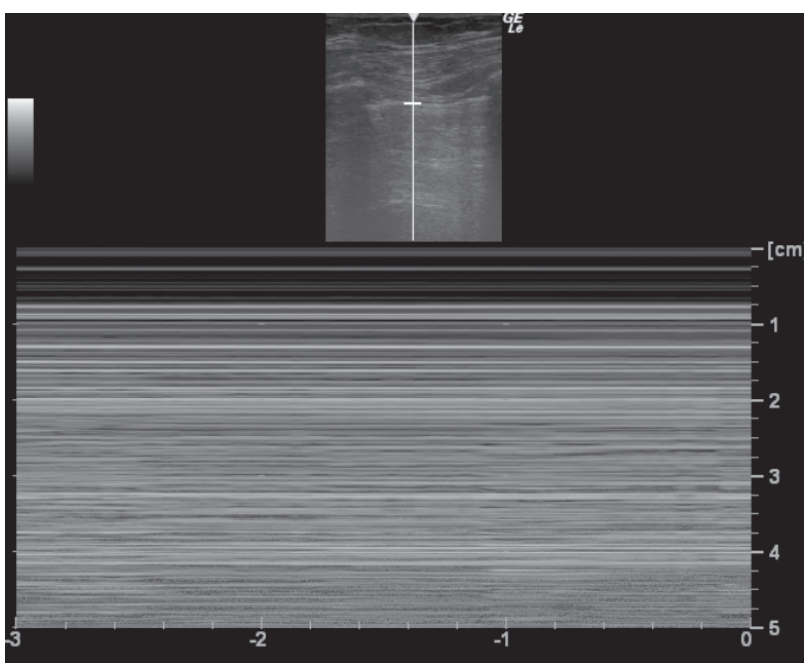

Fig. 5. Pneumothorax on M-mode: stratosphere sign. Below the pleural line, the normal sandy pattern is replaced by horizontal lines showing the absence of lung sliding.

thorax and allows confirmation of pneumothorax with $100 \%$ specificity.[15]

The extension of the small pneumothorax is difficult to assess using CXR in critically ill patients in a supine position. Occasionally, the position of the lung point can help evaluate the pneumothorax extension. The more lateral the lung point on the chest wall, the greater the pneumothorax extension.[16]

\section{2) Interstitial syndrome}

In interstitial syndrome, aeration is impaired due to an increase in interstitial fluid, while some lung aeration is preserved.[17] If B-lines are focally observed, pneumonia/ pneumonitis, atelectasis, pulmonary contusion, pulmonary infarction, or neoplasm should be considered. If multiple diffuse B-lines are observed, pulmonary edema of various causes, interstitial pneumonia/pneumonitis, or diffuse parenchymal lung disease should be considered.[3]

When diffuse multiple B-lines are observed, a few sonographic findings suggest permeability edema rather than cardiogenic pulmonary edema. Pleural line irregularity, reduction or absence of lung sliding and alveolar consolidations with spared areas are predictive findings of permeability edema.[18]

\section{3) Monitoring lung disease}

Because the number of B-lines represents the degree 
Table 2. Lung ultrasound score

\begin{tabular}{llc}
\hline Pattern & \multicolumn{1}{c}{ Charateristics } & Points \\
\hline N & Normal aeration or less than 2 isolated B-lines & 0 \\
B1 & Moderate loss of aeration with multiple, well-defined B-lines & 1 \\
B2 & Severe loss of aeration with multiple coalescent B-lines & 2 \\
C & Lung consolidation & 3 \\
\hline
\end{tabular}

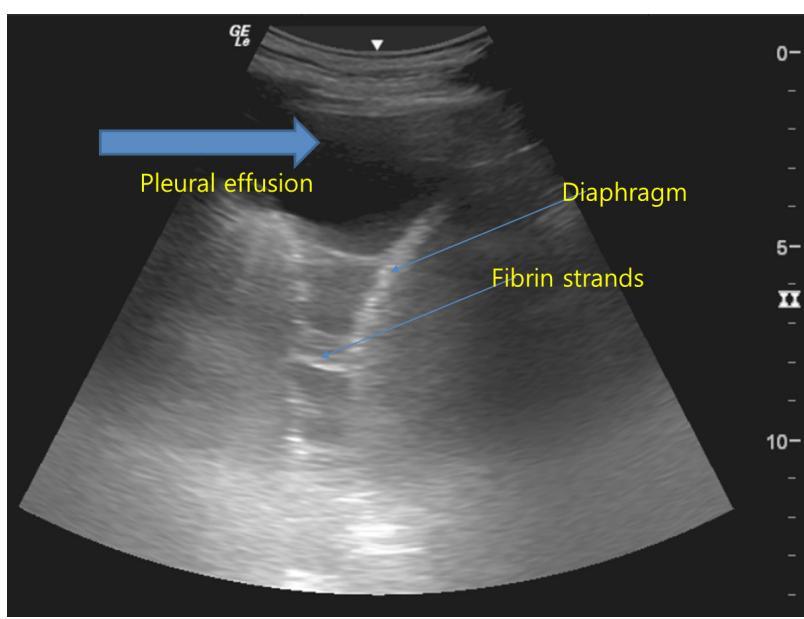

Fig. 6. Sonographic appearance of pleural effusion. Fibrin strands are seen floating inside anechoic pleural effusions suggestive of exudative pleural effusion.

of lung aeration loss, LUS can be used to monitor these changes. LUS has been used to monitor aeration changes and the effects of therapy in acute pulmonary edema, ARDS and pneumonia.[7] Also, LUS has been used as a monitoring tool to assess the response to recruitment maneuvers in critically ill patients.[19] A semiquantitative scoring system of 4 ultrasound aeration patterns has been suggested (Table 2). $[7,20]$

\section{4) Pleural effusion}

Pleural effusion is usually seen on LUS as an anechoic or hypoechoic space between the visceral and parietal pleura (Fig. 6). In supine patients, the optimal site for evaluation is at the posterior axillary line above the diaphragm. In this position, diaphragmatic movement, which is the swaying of the atelectatic lung within the pleural effusion, can be visualized.

The nature of the pleural effusion, whether transudate or exudate, cannot be accurately predicted using LUS. However, a few signs can be helpful. Transudates are anechoic and homogenous echogenic effusions suggest hemorrhagic effusions or empyema.[21] Septated fluids are usually observed in infected exudates and in malignant effusions. LUS also helps guide bedside thoracentesis in patients receiving mechanical ventilation.[22]

\section{Limitations}

Clinicians using LUS in the ICU should be aware of its limitations. Ultrasound is highly operator dependent. The operator must be accurately trained to obtain correct images and adequately interpret them. Finding an adequate acoustic window can be difficult in patients with obesity, thoracic dressings, subcutaneous emphysema or pleural calcifications. Though LUS is useful for assessing peripheral lung parenchymal lesions, assessing central lesions is difficult if they are surrounded by aerated lung.

\section{Conclusion}

Bedside LUS allows fast, accurate, real-time bedside examination of critically ill patients in the ICU. Intensivists should become familiar with the use of LUS as a diagnostic and a monitoring tool.

\section{ORCID}

Jinwoo Lee

http://orcid.org/0000-0003-0958-106X

\section{References}

1) Warren J, Fromm RE Jr, Orr RA, Rotello LC, Horst HM; American College of Critical Care Medicine: Guidelines for the inter- and intrahospital transport of critically ill patients. Crit Care Med 2014; 32: 256-62. 
2) Mayo JR, Aldrich J, Muller NL; Fleischner Society: Radiation exposure at chest CT: a statement of the Fleischner Society. Radiology 2003; 228: 15-21.

3) Volpicelli G, Elbarbary M, Blaivas M, Lichtenstein DA, Mathis G, Kirkpatrick AW, et al: International evidence-based recommendations for point-of-care lung ultrasound. Intensive Care Med 2012; 38: 577-91.

4) Volpicelli G, Mussa A, Garofalo G, Cardinale L, Casoli $\mathrm{G}$, Perotto F, et al: Bedside lung ultrasound in the assessment of alveolar-interstitial syndrome. Am J Emerg Med 2006; 24: 689-96.

5) Lichtenstein DA, Mezière GA: Relevance of lung ultrasound in the diagnosis of acute respiratory failure: the BLUE protocol. Chest 2008; 134: 117-25.

6) Bouhemad B, Zhang M, Lu Q, Rouby JJ: Clinical review: bedside lung ultrasound in critical care practice. Crit Care 2007; 11: 205.

7) Via G, Storti E, Gulati G, Neri L, Mojoli F, Braschi A: Lung ultrasound in the ICU: from diagnostic instrument to respiratory monitoring tool. Minerva Anestesiol 2012; 78: 1282-96.

8) Baldi G, Gargani L, Abramo A, D’Errico L, Caramella D, Picano E, et al: Lung water assessment by lung ultrasonography in intensive care: a pilot study. Intensive Care Med 2013; 39: 74-84.

9) Via G, Lichtenstein D, Mojoli F, Rodi G, Neri L, Storti E, et al: Whole lung lavage: a unique model for ultrasound assessment of lung aeration changes. Intensive Care Med 2010; 36: 999-1007.

10) Lichtenstein D, Mézière G, Biderman $P$, Gepner $A$, Barré $\mathrm{O}$ : The comet-tail artifact. An ultrasound sign of alveolar-interstitial syndrome. Am J Respir Crit Care Med 1997; 156: 1640-6.

11) Lichtenstein D, Mezière G, Seitz J: The dynamic air bronchogram. A lung ultrasound sign of alveolar consolidation ruling out atelectasis. Chest 2009; 135: 1421-5.
12) Lichtenstein DA, Menu Y: A bedside ultrasound sign ruling out pneumothorax in the critically ill. Lung sliding. Chest 1995; 108: 1345-8.

13) Lichtenstein DA, Mezière G, Lascols N, Biderman $P$, Courret JP, Gepner A, et al: Ultrasound diagnosis of occult pneumothorax. Crit Care Med 2005; 33: 1231-8.

14) Husain LF, Hagopian L, Wayman D, Baker WE, Carmody KA: Sonographic diagnosis of pneumothorax. J Emerg Trauma Shock 2012; 5: 76-81.

15) Lichtenstein D, Mezière G, Biderman P, Gepner A: The "lung point": an ultrasound sign specific to pneumothorax. Intensive Care Med 2000; 26: 1434-40.

16) Volpicelli G: Sonographic diagnosis of pneumothorax. Intensive Care Med 2011; 37: 224-32.

17) Volpicelli G: Lung sonography. J Ultrasound Med 2013; 32: 165-71.

18) Copetti R, Soldati G, Copetti P: Chest sonography: a useful tool to differentiate acute cardiogenic pulmonary edema from acute respiratory distress syndrome. Cardiovasc Ultrasound 2008; 6: 16

19) Bouhemad B, Brisson H, Le-Guen M, Arbelot C, Lu Q, Rouby JJ: Bedside ultrasound assessment of positive end-expiratory pressure-induced lung recruitment. Am J Respir Crit Care Med 2011; 183: 341-7.

20) Soummer A, Perbet S, Brisson H, Arbelot C, Constantin JM, Lu Q, et al: Ultrasound assessment of lung aeration loss during a successful weaning trial predicts postextubation distress*. Crit Care Med 2012; 40: 2064-72.

21) Yang PC, Luh KT, Chang DB, Wu HD, Yu CJ, Kuo SH: Value of sonography in determining the nature of pleural effusion: analysis of 320 cases. AJR Am J Roentgenol 1992; 159: 29-33.

22) Mayo PH, Goltz HR, Tafreshi M, Doelken P: Safety of ultrasound-guided thoracentesis in patients receiving mechanical ventilation. Chest 2004; 125: 1059-62. 